\title{
SINGULAR BOUNDARY VALUE PROBLEM ON INFINITE TIME SCALE
}

\author{
ZHAO-CAI HAO, JIN LIANG, AND TI-JUN XIAO
}

Received 1 June 2005; Accepted 14 August 2005

This paper deals with a class of singular boundary value problems of differential equations on infinite time scale. An existence theorem of positive solutions is established by using the Schauder fixed point theorem and perturbation and operator approximation method, which resolves the singularity successfully and differs from those of some papers. In the end of the paper, an example is given to illustrate our main result.

Copyright (C) 2006 Zhao-Cai Hao et al. This is an open access article distributed under the Creative Commons Attribution License, which permits unrestricted use, distribution, and reproduction in any medium, provided the original work is properly cited.

\section{Introduction}

Recently, much attention is attracted by questions of existence of positive solutions to boundary value problems for differential equations on measure chains, see, for example, $[1-7,10-12,15,17-19,21]$. This has been mainly due to its unification of the theory of differential and difference equations. An introduction to this unification is given in $[5,17,19]$. Now, this study is still a new area of fairly theoretical exploration in mathematics. However, it has led to several important applications, for example, in the study of insect population models, neural networks, heat transfer, and epidemic models, see, for example, [1].

To the author's knowledge, very little work has been done to the existence of positive solutions on infinite time scale. In 2002 and 2004, [2, 15] considered boundary value problems on infinite time scale. Problems in $[2,15]$ are all nonsingular and the method used is obtaining the local solutions at first and then extending them to the whole interval.

Motivated by works of $[2,14,15,21]$, in this paper, we investigate the existence of positive solutions to the following singular boundary value problem on infinite time scale

$$
\begin{gathered}
x^{\Delta \Delta}(t)-k^{2} x(\sigma(t))+m(t) f(t, x(\sigma(t)))=0, \quad t \in \mathbb{R}^{+}, \\
x(0)=0, \quad \lim _{t \rightarrow \infty} x(t)=0,
\end{gathered}
$$

Hindawi Publishing Corporation

Discrete Dynamics in Nature and Society

Volume 2006, Article ID 71580, Pages 1-13

DOI 10.1155/DDNS/2006/71580 
2 Singular boundary value problem on infinite time scale

where $k>0$ is given, $\mathbb{R}^{+}:=[0, \infty), m(\cdot)$ and $f(\cdot, \cdot)$ are given functions. $m(t)$ may be singular at $t=0$ and $f(t, x)$ may be singular at $x=0$.

To understand the notation used above and the ideas of time scale, some preliminary definitions are useful.

Definition 1.1. A time scale $\mathbb{T}$ is a nonempty closed subset of $\mathbb{R}$, the set of real numbers.

Throughout this paper, the assumption is made that $\mathbb{T}$ has the subspace topology inherited from the standard topology on $\mathbb{R}$. We also assume that there exist $t_{n}^{1}, t_{n}^{2} \in \mathbb{R}^{+}$, $n \in\{1,2,3, \ldots\} \equiv \mathbb{N}$ such that

$$
t_{n}^{1} \uparrow \infty \quad \text { as } n \longrightarrow \infty, \quad t_{n}^{2} \downarrow 0 \quad \text { as } n \longrightarrow \infty .
$$

Definition 1.2. Define the interval in $\mathbb{T}$ :

$$
[a, b]:=\{t \in \mathbb{T} \text { such that } a \leq t \leq b\} .
$$

Other type of intervals are defined similarly.

A time scale may or may not be connected, so the concept of jump operators is useful. Definition 1.3. Define the forward jump operator (resp., backward jump operator) at $t$ for $t<\sup \mathbb{\mathbb { T }}$ (resp., for $t>\inf \mathbb{T}$ ) by

$$
\sigma(t):=\inf \{\tau>t ; \tau \in \mathbb{T}\} \in \mathbb{T} \quad(\text { resp., } \rho(t):=\sup \{\tau<t ; \tau \in \mathbb{T}\} \in \mathbb{T})
$$

for all $t \in \mathbb{T}$.

In this definition, we put $\inf \phi=\sup \mathbb{T}$ (i.e., $\sigma(M)=M$ if $\mathbb{T}$ has a maximum $M$ ) and $\sup \phi=\inf \mathbb{T}$ (i.e., $\rho(m)=m$ if $\mathbb{T}$ has a minimum $m$ ), where $\phi$ denotes the empty set. If $\sigma(t)>t$, we say $t$ is right scattered, while if $\rho(t)<t$ we say that $t$ is left scattered. Points that are right scattered and left scattered at the same time are called isolated. Also, if $t<\sup \mathbb{T}$ and $\sigma(t)=t$, then $t$ is called right-dense, and if $t>\inf \mathbb{T}$ and $\rho(t)=t$, then $t$ is called leftdense. Points that are right-dense and left-dense are called dense. If $\mathbb{T}$ has a left-scattered maximum $M$, then we define $\mathbb{T}^{*}:=\mathbb{T}-\{M\}$, otherwise $\mathbb{T}^{*}:=\mathbb{T}$.

Now we consider a function $x: \mathbb{T} \rightarrow \mathbb{R}$ and define so-called delta (Hilger) derivative of $x$ at a point $t \in \mathbb{T}^{*}$.

Definition 1.4. Assume $x: \mathbb{T} \rightarrow \mathbb{R}$ and fix $t \in \mathbb{T}^{*}$. Then define $x^{\triangle}(t)$ to be the number (provided that it exists) with the property that given any $\varepsilon>0$, there is a neighborhood $U$ of $t$ such that

$$
\left\|[x(\sigma(t))-x(s)]-x^{\triangle}(t)[\sigma(t)-s]\right\| \leq \varepsilon|\sigma(t)-s|,
$$

for all $s \in U$. Call $x^{\triangle}(t)$ the delta (or Hilger) derivative of $x(t)$ at $t \in \mathbb{T}$. The derivative can also be defined in terms of a limit as follows:

$$
x^{\triangle}(t):=\lim _{s \rightarrow t, \sigma(s) \neq t} \frac{x(\sigma(s))-x(t)}{\sigma(s)-t}=\lim _{s \rightarrow t, \sigma(t) \neq s} \frac{x(\sigma(t))-x(s)}{\sigma(t)-s} .
$$

The second derivative of $x(t)$ is defined by $x^{\triangle} \triangle(t)=\left(x^{\triangle}\right)^{\triangle}(t)$. 
Reference [5, Theorem 1.3(iv)] tells us that if $t \in \mathbb{T}^{*}$ and $f: \mathbb{T} \rightarrow \mathbb{R}^{+}$is delta-differential at $t$, then

$$
f(\sigma(t))=f(t)+(\sigma(t)-t) f^{\Delta}(t)
$$

Definition 1.5. A function $F: \mathbb{T} \rightarrow \mathbb{R}$ is called delta-antiderivative of $f: \mathbb{T} \rightarrow \mathbb{R}$ provided that $F^{\triangle}(t)=f(t)$ holds for all $t \in \mathbb{T}^{*}$. In this case, define the integral of $f$ by

$$
\int_{a}^{t} f(\tau) \triangle \tau=F(t)-F(a)
$$

It is important to make further illumination on the integral in Definition 1.5. We get from [5, Theorem 1.30] that

$$
\int_{a}^{b} f(t) \Delta t= \begin{cases}\sum_{t \in[a, b)}[\sigma(t)-t] f(t) & \text { if } a<b, \\ 0 & \text { if } a=b \\ -\sum_{t \in[b, a)}[\sigma(t)-t] f(t) & \text { if } a>b\end{cases}
$$

where $[a, b]$ consists of only isolated points and $f \in \mathbb{C}_{\mathrm{rd}}[a, b]$. [5, Chapter 5, pages 145$155]$ investigated improper integrals on time scales and presented that

$$
\int_{a}^{\infty} f(t) \Delta t=\lim _{A \rightarrow \infty}\left\{\int_{a}^{A} f(t) \Delta t\right\}
$$

if the integral $F(A)=\int_{a}^{A} f(t) \Delta t$ approaches a finite limit as $A \rightarrow \infty$. We call that limit the improper integral of first kind of $f$ from $a$ to $\infty$. In such a case we say that the improper integral $\int_{a}^{\infty} f(t) \Delta t$ exists or that it is convergent. If $f$ has a singularity at $t=b$ and the left-sided limit $\lim _{c \rightarrow b^{-}} \int_{a}^{c} f(t) \Delta t$ exists as a finite number, then we say that the improper integral $\int_{a}^{b} f(t) \Delta t$ exists or that it is convergent. In such a case we call this limit the value of the improper integral $\int_{a}^{b} f(t) \Delta t$ and write

$$
\int_{a}^{b} f(t) \Delta t=\lim _{c \rightarrow b^{-}} \int_{a}^{c} f(t) \Delta t
$$

Proposition 1.6 [5, Theorem 5.52]. Let the inequalities $0 \leq f(t) \leq g(t)$ be satisfied for all $t \in[a, \infty)$. Then the convergence of the improper integral

$$
\int_{a}^{\infty} g(t) \Delta t
$$

implies the improper integral

$$
\int_{a}^{\infty} f(t) \Delta t
$$


4 Singular boundary value problem on infinite time scale

and the inequality

$$
\int_{a}^{\infty} f(t) \Delta \leq \int_{a}^{\infty} g(t) \Delta
$$

while the divergence of integral (1.13) implies the divergence of integral (1.12).

Formulas (1.9)-(1.11) and the comparison test Proposition 1.6 will play an important role in Example 4.1.

Definition 1.7. A function $f: \mathbb{T} \rightarrow \mathbb{R}$ is called rd-continuous provided it is continuous at right-dense points in $\mathbb{T}$ and its left-sided limits exist (finite) at left-dense points in $\mathbb{T}$. The set of rd-continuous functions $f: \mathbb{T} \rightarrow \mathbb{R}$ will be denoted by

$$
C_{\mathrm{rd}}=C_{\mathrm{rd}}(\mathbb{T})=C_{\mathrm{rd}}(\mathbb{T}, \mathbb{R})
$$

Definition 1.8. Define $\mathbb{S}$ to be the set of all functions $x: \mathbb{T} \rightarrow \mathbb{R}$ such that

$$
\mathbb{S}:=\left\{x: x \in C\left(\mathbb{R}^{+} ; \mathbb{R}\right), x^{\Delta \Delta} \in C\left(\mathbb{R}^{+} ; \mathbb{R}\right)\right\}
$$

A positive solution to BVP (1.1) is a function $x \in \mathbb{S}$ which satisfies (1.1) for each $t \in \mathbb{R}^{+}$ and $x(t)>0$ on $(0, \infty)$.

The aim of this paper is to give the existence of positive solutions of BVP (1.1) on infinite time scale $\mathbb{R}^{+}$. Here the problem (1.1) may be singular. Generally speaking, limit conditions are required on many researches on time scale at present and the Krasnosel'skii fixed point theorem is often used also, see, for example, $[3,6-13,16,18,20]$. By using the Schauder fixed point theorem (the Krasnosel'skii fixed point theorem does not applies to this paper) and monotone conditions (see $\left(\mathrm{H}_{1}\right)$ ), not limit conditions, we obtain directly the global positive solution of BVP (1.1). Perturbation and operator approximation method are used to resolve the singularity. Moreover, some compactness criterion (see Proposition 2.1) will help us to resolve the new problem that is how to prove the completely continuity of an operator (see (3.1)) defined on the infinite time scale $\mathbb{R}^{+}$. These are the differences between this paper and papers [2, 3, 6-16, 18, 20, 21].

We organize this paper as follows. In Section 2, starting with some preliminary results, we then state our main result (see Theorem 2.2), an existence theorem of positive solutions of BVP (1.1). Section 3 concentrates on the proof of Theorem 2.2. An example is given to illustrate Theorem 2.2 in Section 4.

\section{Preliminaries and main result}

We will first present some properties of the Green's function for the following homogeneous BVP:

$$
\begin{gathered}
x^{\Delta \Delta}(t)-k^{2} x(\sigma(t))=0, \quad t \in \mathbb{R}^{+}, \\
x(0)=0, \quad \lim _{t \rightarrow \infty} x(t)=0 .
\end{gathered}
$$


The Green function for BVP (2.1) is given as follows:

$$
G(t, s)=\frac{1}{2 k} \begin{cases}e^{-k \sigma(s)}\left(e^{k t}-e^{-k t}\right), & 0 \leq t \leq s, \\ e^{-k t}\left(e^{k \sigma(s)}-e^{-k \sigma(s)}\right), & 0 \leq \sigma(s) \leq t .\end{cases}
$$

Obviously,

$$
\begin{gathered}
G(t, s) \geq 0, \quad(t, s) \in \mathbb{R}^{+} \times \mathbb{R}^{+} . \\
e^{-\mu t} G(t, s) \leq e^{-k \sigma(s)} G(\sigma(s), s), \quad \mu \geq k,(t, s) \in \mathbb{R}^{+} \times \mathbb{R}^{+} .
\end{gathered}
$$

Define

$$
\mathbb{E}:=\left\{x \in C\left(\mathbb{R}^{+}\right): \sup _{t \in \mathbb{R}^{+}}\left\{|x(t)| e^{-\lambda^{*} t}\right\}<\infty\right\}
$$

where

$$
\lambda^{*}>k \text { is given. }
$$

It is easy to testify that $\mathbb{E}$ is a Banach space equipped with the following norm:

$$
\|x\|:=\sup _{t \in \mathbb{R}^{+}}\left\{|x(t)| e^{-\lambda^{*} t}\right\} .
$$

The Schauder fixed point theorem will be used in this paper. So we need also the following modification of the compactness criterion.

Proposition 2.1 (cf. [22]). Let $\mathbb{E}_{1}$ be the space given by

$$
\mathbb{E}_{1}:=\left\{x \in C\left(\mathbb{R}^{+}\right): \sup _{t \in \mathbb{R}^{+}}\{|x(t)| p(t)\}<\infty\right\}
$$

equipped with the norm

$$
\|x\|:=\sup _{t \in \mathbb{R}^{+}}\{|x(t)| p(t)\}
$$

where $p: \mathbb{R}^{+} \rightarrow(0, \infty)$ is a continuous function. Let $\Omega \subset \mathbb{E}_{1}$. If the functions $x \in \Omega$ are almost equicontinuous on $\mathbb{R}^{+}$(i.e., they are equicontinuous in each interval $[0, T], 0<T<\infty$ ) and uniformly bounded in the sense of the norm

$$
\|x\|_{q}:=\sup _{t \in \mathbb{R}^{+}}\{|x(t)| q(t)\},
$$

where the function $q$ is positive and continuous on $\mathbb{R}^{+}$and

$$
\lim _{t \rightarrow \infty} \frac{p(t)}{q(t)}=0,
$$

then $\Omega$ is relatively compact in $\mathbb{E}_{1}$. 
6 Singular boundary value problem on infinite time scale

Now, let us present the main result of this paper as follows.

Theorem 2.2. Assume the following conditions hold:

$\left(\mathrm{H}_{1}\right) f(t, x): \mathbb{R}^{+} \times(0, \infty) \rightarrow R^{+}$is continuous ( $f$ may be singular at $x=0$ ) and $f(t, x)$ is decreasing in relative to $x$ and satisfies also

$$
\sup _{(t, x) \in \mathbb{R}^{+} \times \mathbb{R}^{+}} f(t, x)<\infty
$$

$\left(\mathrm{H}_{2}\right) m(\cdot):(0, \infty) \rightarrow R^{+}$is $r d$-continuous and may be singular at $t=0$;

$\left(\mathrm{H}_{3}\right) \int_{0}^{\infty} e^{-k \sigma(s)} G(\sigma(s), s) m(s) \triangle s<\infty$ hold all. Then, BVP (1.1) has at least one positive solution.

\section{Proof of Theorem 2.2}

The idea of the proof is that we prove first that the following BVP $(1.1)_{\varepsilon}$, the perturbation of BVP (1.1), has at least one positive solution for any $\varepsilon$. Then we prove that the set of those positive solutions has a convergent subsequence and its limit is just a positive solution of BVP (1.1).

Definition 3.1. If $x(t) \in C^{2}((0, \infty)) \cap C\left(R^{+}\right)$satisfies

(1) $x(t)=\varepsilon+\int_{0}^{\infty} G(t, s) m(s) f(s, x(\sigma(s))) \Delta s$;

(2) $x^{\Delta \Delta}(t)-k^{2} x(\sigma(t))+m(t) f(t, x(\sigma(t)))=0$ and $x(t)>0, t \in(0, \infty)$, then call it a positive solution of the following BVP $(1.1)_{\varepsilon}$ :

$$
\begin{gathered}
x^{\Delta \Delta}(t)-k^{2} x(\sigma(t))+m(t) f(t, x(\sigma(t)))=0, \\
x(0)=\varepsilon, \quad \lim _{t \rightarrow \infty} x(t)=\varepsilon,
\end{gathered}
$$

with $\varepsilon \in(0,1)$ a parameter.

Proof of Theorem 2.2. The rather long proof will be divided into three steps.

Step 1. We show that BVP $(1.1)_{\varepsilon}$ has at least one positive solution for any $\varepsilon \in(0,1)$.

Let us first define an operator $A$ as follows

$$
(A x)(t):=\varepsilon+\int_{0}^{\infty} G(t, s) m(s) f(s, x(\sigma(s))) \Delta s, \quad t \in \mathbb{R}^{+}, x \in D_{\varepsilon},
$$

where

$$
D_{\varepsilon}:=\left\{x \in \mathbb{E} ; 0<\varepsilon \leq x(t) \leq(A \varepsilon)(t), t \in \mathbb{R}^{+}\right\} .
$$

By conditions $\left(\mathrm{H}_{1}\right)-\left(\mathrm{H}_{3}\right)$ and (2.4) we get, for any $x \in D_{\varepsilon}$ and $t \in \mathbb{R}^{+}$,

$$
\begin{aligned}
|A x(t)| e^{-\lambda^{*} t} & =\varepsilon e^{-\lambda^{*} t}+\int_{0}^{\infty} e^{-\lambda^{*} t} G(t, s) m(s) f(s, x(\sigma(s))) \Delta s \\
& \leq \varepsilon+\int_{0}^{\infty} e^{-k \sigma(s)} G(\sigma(s), s) m(s) f(s, x(\sigma(s))) \Delta s \\
& \leq 1+\sup _{(u, v) \in \mathbb{R}^{+} \times \mathbb{R}^{+}} f(u, v) \int_{0}^{\infty} e^{-k \sigma(s)} G(\sigma(s), s) m(s) \Delta s .
\end{aligned}
$$


Equation (3.3) implies that

$$
\sup _{t \in \mathbb{R}^{+}}\left\{|A x(t)| e^{-\lambda^{*} t}\right\} \leq 1+\sup _{(u, x v) \in \mathbb{R}^{+} \times R^{+}} f(u, v) \int_{0}^{\infty} e^{-k \sigma(s)} G(\sigma(s), s) m(s) \Delta s<\infty .
$$

This implies that the operator $A$ is well defined and $A x \in \mathbb{E}, \forall x \in D_{\varepsilon}$.

Clearly, fixed points of operator $A$ in $D_{\varepsilon}$ are also positive solutions of BVP $(1.1)_{\varepsilon}$. So in order to use the well-known Schauder fixed point theorem to obtain fixed point of operator $A$, now, let us concentrate on the proof that $A: D_{\varepsilon} \rightarrow D_{\varepsilon}$ is completely continuous. Firstly, it is easy to see that $A\left(D_{\varepsilon}\right) \subset D_{\varepsilon}$. Secondly, we claim that $A: D_{\varepsilon} \rightarrow D_{\varepsilon}$ is completely continuous. We can see also that the singularity of $f$ at $x=0$ has been removed successfully by using the perturbation. The singularity of $m$ at $t=0$ will be solved also by using approximation method. For further details, there are two cases to be considered.

Case 1. If $m(t) \in C[0, \infty)$ holds in $\left(\mathrm{H}_{2}\right)$, let us prove that $A$ is completely continuous. For any $x \in D_{\varepsilon}, t \in \mathbb{R}^{+}$and $\lambda_{1}^{*}$ such that

$$
k<\lambda_{1}^{*}<\lambda^{*}
$$

we see similarly from the proof of (3.3) that functions $\left\{A x: x \in D_{\varepsilon}\right\}$ are uniformly bounded with respect to the norm

$$
\|x\|_{\lambda_{1}^{*}}:=\sup _{t \in \mathbb{R}^{+}}\left\{|x(t)| e^{-\lambda_{1}^{*} t}\right\}
$$

Moreover, for any $N \in(0, \infty)$, the fact that

$$
G(t, s) \in C\left([0, N] \times \mathbb{R}^{+}\right), \quad m(t) \in C([0, N]), \quad f(t, x) \in C\left([0, N] \times D_{\varepsilon}\right)
$$

and standard arguments tell us that $\left\{A x: x \in D_{\varepsilon}\right\}$ are equicontinuous in interval $[0, N]$. So $\left\{A x: x \in D_{\varepsilon}\right\}$ are almost equicontinuous on $\mathbb{R}^{+}$. Thus, if we set

$$
p(t):=e^{-\lambda^{*} t}, \quad q(t):=e^{-\lambda_{1}^{*} t},
$$

then Proposition 2.1 implies that $\left\{A x: x \in D_{\varepsilon}\right\}$ is a precompact set in $\mathbb{E}$. Hence, $A$ is completely continuous.

Case 2. We claim that the operator $A$ given by (3.1) is completely continuous when $m(t)$ is singular at $t=0$. Choose $\left\{\eta_{n}\right\}_{n=1}^{\infty} \subset \mathbb{R}^{+}$such that $\eta_{n} \downarrow 0$ as $n \rightarrow \infty$. Define operator sequences $\left\{A_{n}\right\}$ by

$$
\left(A_{n} x\right)(t)=\varepsilon+\int_{\eta_{n}}^{\infty} G(t, s) m(s) f(s, x(\sigma(s))) \Delta s, \quad x \in D_{\varepsilon}, t \in \mathbb{R}^{+}, n \geq 1 .
$$

It follows from the proof of Case 1 that

$$
A_{n}: D_{\varepsilon} \longrightarrow D_{\varepsilon} \text { is completely continuous for each } n \geq 1 \text {. }
$$


8 Singular boundary value problem on infinite time scale

By condition $\left(\mathrm{H}_{1}\right),(2.4)$, and (2.6), we have

$$
\begin{aligned}
\left|(A x)(t)-\left(A_{n} x\right)(t)\right| e^{-\lambda^{*} t} & =\int_{0}^{\eta_{n}} e^{-\lambda^{*} t} G(t, s) m(s) f(s, x(\sigma(s))) \Delta s \\
& \leq \int_{0}^{\eta_{n}} e^{-k \sigma(s)} G(\sigma(s), s) m(s) \Delta s \sup _{(u, v) \in \mathbb{R}^{+} \times \mathbb{R}^{+}} f(u, v),
\end{aligned}
$$

and so

$$
\sup _{t \in \mathbb{R}^{+}}\left\{\left|(A x)(t)-\left(A_{n} x\right)(t)\right| e^{-\lambda^{*} t}\right\} \leq \sup _{(u, v) \in \mathbb{R}^{+} \times R^{+}} f(u, v) \int_{0}^{\eta_{n}} e^{-k \sigma(s)} G(\sigma(s), s) m(s) \Delta s .
$$

Condition $\left(\mathrm{H}_{3}\right)$ and the absolute continuity of integral imply that

$$
\lim _{n \rightarrow \infty} \int_{0}^{\eta_{n}} e^{-k \sigma(s)} G(\sigma(s), s) m(s) \Delta s=0 .
$$

Equations (3.10), (3.12), and (3.13) justify that operator $A$ is completely continuous.

Consequently, we know from the Schauder fixed point theorem that the operator $A$ has one fixed point in $D_{\varepsilon}$. Without loss of generality, we write it as $x(t, \varepsilon)$. Then we may compute that

$$
\begin{gathered}
x(t, \varepsilon)=\varepsilon+\int_{0}^{\infty} G(t, s) m(s) f(s, x(\sigma(s), \varepsilon)) \Delta s \\
x^{\Delta \Delta}(t, \varepsilon)-k^{2} x(\sigma(t), \varepsilon)+m(t) f(t, x(\sigma(t), \varepsilon))=0, \quad x(t, \varepsilon)>0, t \in(0, \infty) .
\end{gathered}
$$

So $x(t, \varepsilon)$ is a positive solution of BVP $(1.1)_{\varepsilon}$.

Step 2. Let us show that if $1>\varepsilon_{1}>\varepsilon_{2}>0$, then

$$
0 \leq x\left(t, \varepsilon_{1}\right)-x\left(t, \varepsilon_{2}\right) \leq \varepsilon_{1}-\varepsilon_{2}, \quad \forall t \in \mathbb{R}^{+} .
$$

For any $1>\varepsilon_{1}>\varepsilon_{2}>0$, we show first that

$$
0 \leq x\left(t, \varepsilon_{1}\right)-x\left(t, \varepsilon_{2}\right), \quad \forall t \in \mathbb{R}^{+} .
$$

If not, then either

$$
x\left(t, \varepsilon_{1}\right)<x\left(t, \varepsilon_{2}\right), \quad \forall t \in \mathbb{R}^{+},
$$

or

$$
x\left(t, \varepsilon_{1}\right)<x\left(t, \varepsilon_{2}\right), \quad t \in S_{1}, \quad x\left(t, \varepsilon_{1}\right) \geq x\left(t, \varepsilon_{2}\right), \quad t \in S_{2},
$$

holds, where $S_{1}$ and $S_{2}$ are some nonempty subsets of $\mathbb{R}^{+}$. If (3.17) holds, then for any $t \in \mathbb{R}^{+}$, we have from (3.14) that

$$
\varepsilon_{1}+\int_{0}^{\infty} G(t, s) m(s) f\left(s, x\left(\sigma(s), \varepsilon_{1}\right)\right) \Delta s<\varepsilon_{2}+\int_{0}^{\infty} G(t, s) m(s) f\left(s, x\left(\sigma(s), \varepsilon_{2}\right)\right) \Delta s .
$$


Then (3.17), (3.19) and the monotone property of $f$ imply that

$$
0 \leq \int_{0}^{\infty} G(t, s) m(s)\left[f\left(s, x\left(\sigma(s), \varepsilon_{1}\right)\right)-f\left(s, x\left(\sigma(s), \varepsilon_{2}\right)\right)\right] \Delta s<\varepsilon_{2}-\varepsilon_{1}<0,
$$

a contradiction. If (3.18) holds, we write $W(t):=x\left(t, \varepsilon_{2}\right)-x\left(t, \varepsilon_{1}\right), t \in \mathbb{R}^{+}$. Then the continuity of $W(t)$ and (3.18) tell us that there exists a nonempty and closed $S_{3} \subset \mathbb{R}^{+}$such that $W(t)$ is sign-changing on $S_{3}$. Therefore,

$$
W(t) \not \equiv \text { constant }, \quad t \in S_{3} \text {. }
$$

Set

$$
W\left(t_{0}\right)=\max _{t \in S_{3}} W(t)
$$

Clearly, $W\left(t_{0}\right)>0$ and $t_{0}$ is an extreme value point tells us that $W^{\Delta}\left(t_{0}\right)=0$. Then we know from (1.7) that

$$
W\left(\sigma\left(t_{0}\right)\right)=W\left(t_{0}\right)+W^{\Delta}\left(t_{0}\right)\left(\sigma\left(t_{0}\right)-t_{0}\right)>0 .
$$

Furthermore, (3.21) and (3.22) imply that $t_{0}$ is not minimal value point. This gives

$$
W^{\Delta \Delta}\left(t_{0}\right) \leq 0 .
$$

On the other hand, (3.23) gives $x\left(\sigma\left(t_{0}\right), \varepsilon_{1}\right)-x\left(\sigma\left(t_{0}\right), \varepsilon_{2}\right)<0$. So we know from the property of $f(t, x)$ that

$$
f\left(t_{0}, x\left(\sigma\left(t_{0}\right), \varepsilon_{1}\right)\right)-f\left(t_{0}, x\left(\sigma\left(t_{0}\right), \varepsilon_{2}\right)\right) \geq 0 .
$$

Hence

$$
\begin{aligned}
W^{\Delta \Delta}\left(t_{0}\right) & =x^{\Delta \Delta}\left(t_{0}, \varepsilon_{2}\right)-x^{\Delta \Delta}\left(t_{0}, \varepsilon_{1}\right) \\
& =k^{2} W\left(\sigma\left(t_{0}\right)\right)+m\left(t_{0}\right)\left[f\left(t_{0}, x\left(\sigma\left(t_{0}\right), \varepsilon_{1}\right)\right)-f\left(t_{0}, x\left(\sigma\left(t_{0}\right), \varepsilon_{2}\right)\right)\right] \\
& \geq k^{2} W\left(\sigma\left(t_{0}\right)\right) .
\end{aligned}
$$

Clearly, (3.23) implies that (3.24) contradicts (3.26). So (3.18) does not holds. Consequently, (3.16) holds.

From (3.16), we know

$$
f\left(t, x\left(t, \varepsilon_{1}\right)\right)-f\left(t, x\left(t, \varepsilon_{2}\right)\right) \leq 0, \quad \forall t \in \mathbb{R}^{+} .
$$

Thus, for any $t \in \mathbb{R}^{+}$, we have

$$
x\left(t, \varepsilon_{1}\right)-x\left(t, \varepsilon_{2}\right)=\varepsilon_{1}-\varepsilon_{2}+\int_{0}^{\infty} G(t, s) m(s)\left[f\left(t, x\left(t, \varepsilon_{1}\right)\right)-f\left(t, x\left(t, \varepsilon_{2}\right)\right)\right] \Delta s \leq \varepsilon_{1}-\varepsilon_{2} .
$$

Hence the right-hand side of the inequality (3.15) also holds. 
10 Singular boundary value problem on infinite time scale

Step 3. Steps 1 and 2 imply that there exist one sequence $\left\{\varepsilon_{j}: j=1,2,3, \ldots\right\}$ and one function, without loss of generality, we denote it by $x(t, 0)$, such that

$$
\lim _{j \rightarrow \infty} x\left(t, \varepsilon_{j}\right)=x(t, 0), \quad t \in \mathbb{R}^{+},
$$

uniformly. Let

$$
\begin{gathered}
F_{j}(t, s)=G(t, s) m(s) f\left(s, x\left(\sigma(s), \varepsilon_{j}\right)\right), \quad(t, s) \in \mathbb{R}^{+} \times \mathbb{R}^{+}, j=1,2,3, \ldots, \\
F(t, s)=G(t, s) m(s) \sup _{(u, v) \in \mathbb{R}^{+} \times \mathbb{R}^{+}} f(u, v), \quad(t, s) \in \mathbb{R}^{+} \times \mathbb{R}^{+} .
\end{gathered}
$$

Then

$$
\left|F_{j}(t, s)\right| \leq F(t, s), \quad j=1,2,3, \ldots,(t, s) \in \mathbb{R}^{+} \times R^{+} .
$$

Moreover, (2.4) implies that

$$
\begin{aligned}
\left\|\int_{0}^{\infty} F(t, s) \Delta s\right\| & =\sup _{t \in \mathbb{R}^{+}}\left|e^{-\lambda^{*} t} \int_{0}^{\infty} F(t, s) \Delta s\right| \\
& \leq \sup _{t \in \mathbb{R}^{+}} \int_{0}^{\infty} e^{-k \sigma(s)} G(\sigma(s), s) m(s) \Delta s \sup _{(u, v) \in \mathbb{R}^{+} \times \mathbb{R}^{+}} f(u, v) \\
& <\infty .
\end{aligned}
$$

Now, by using control convergence theorem and (3.29), (3.31), and (3.32), we know that

$$
\begin{gathered}
x(t, 0)=\int_{0}^{\infty} G(t, s) m(s) f(s, x(\sigma(s), 0)) \Delta s, \quad t \in \mathbb{R}^{+}, \\
x(0)=0, \quad \lim _{t \rightarrow \infty} x(t)=0 .
\end{gathered}
$$

Thus $x(t, 0)$ is a positive solution of BVP $(1.1)$. This ends the proof.

\section{An example}

We present the following example to illustrate our main result Theorem 2.2.

Example 4.1. Set

$$
\mathbb{T}=\{0,1,2,3, \ldots\} .
$$

Consider the following BVP:

$$
\begin{gathered}
x^{\Delta \Delta}(t)-x(\sigma(t))+\frac{1}{e^{t}-e^{-t}} f(t, x)=0, \quad t \in \mathbb{R}^{+}, \\
x(0)=0, \quad \lim _{t \rightarrow \infty} x(t)=0,
\end{gathered}
$$

where

$$
f(t, x)= \begin{cases}0, & (t, x) \in \mathbb{R}^{+} \times\{0\}, \\ e^{-x}, & (t, x) \in \mathbb{R}^{+} \times(0, \infty) .\end{cases}
$$


We may claim that BVP (4.2) has at least one positive solution $x \in C\left(\mathbb{R}^{+}\right)$. To say this assertion, what should we do is to validate all conditions of Theorem 2.2.

Write

$$
m(t):=\frac{1}{e^{t}-e^{-t}} .
$$

Clearly, $m(t) \in \mathbb{C}_{\mathrm{rd}}(0, \infty)$. However, $m(t)$ is singular at $t=0$. Hence BVP (4.2) is a singular BVP on $\mathbb{R}^{+}$. This is just the condition $\left(\mathrm{H}_{2}\right)$ in Theorem 2.2. Moreover, (4.3) implies that $f$ satisfies the condition $\left(\mathrm{H}_{1}\right)$ in Theorem 2.2. Now let us prove that the condition $\left(\mathrm{H}_{3}\right)$ in Theorem 2.2 holds here. The Green function of the corresponding homogeneous problem of BVP (4.2) is given by

$$
G(t, s)=\frac{1}{2} \begin{cases}e^{-\sigma(s)}\left(e^{t}-e^{-t}\right), & t \leq s \\ e^{-t}\left(e^{\sigma(s)}-e^{-\sigma(s)}\right), & \sigma(s) \leq t .\end{cases}
$$

Then, for any $n_{0} \in\{1,2,3, \ldots\}$, we compute

$$
\begin{aligned}
\int_{0}^{\infty} e^{-\sigma(s)} G(\sigma(s), s) m(s) \Delta s & =\frac{1}{2} \int_{0}^{\infty} e^{-2 s-2} \frac{\left[e^{s+1}-e^{-s-1}\right]}{e^{s}-e^{-s}} \Delta s \\
& =\frac{1}{2}\left\{\int_{0}^{n_{0}} e^{-2 s-2} \frac{\left[e^{s+1}-e^{-s-1}\right]}{e^{s}-e^{-s}} \Delta s+\int_{n_{0}}^{\infty} e^{-2 s-2} \frac{\left[e^{s+1}-e^{-s-1}\right]}{e^{s}-e^{-s}} \Delta s\right\} \\
& =\frac{1}{2}\left(I_{1}+I_{2}\right) .
\end{aligned}
$$

Set

$$
f_{1}(s):=e^{-2 s-2} \frac{\left[e^{s+1}-e^{-s-1}\right]}{e^{s}-e^{-s}}, \quad f_{2}(s):=\frac{1}{2} e^{-s-1}, \quad t \in \mathbb{R}^{+} .
$$

Then we have for any $s \in(0, \infty)$,

$$
f_{1}(s) \leq e^{-2 s-2} \frac{e^{2 s+2}-1}{e^{s}-e^{-s}} \leq e^{-2 s-2} \frac{e^{2 s+1} e}{e^{s}-e^{-s}} \leq \frac{1}{2 \sqrt{e^{2 s+1} e}}=\frac{1}{2} e^{-s-1}=f_{2}(s) .
$$

Formulas (1.9) and (1.10) help us to obtain that

$$
\begin{gathered}
\int_{0}^{n_{0}} f_{2}(s) \Delta s=\frac{1}{2} \sum_{s \in\left[0, n_{0}-1\right]} e^{-s-1}=\frac{1-e^{-n_{0}}}{2(e-1)}<\frac{1}{e-1} . \\
\int_{n_{0}}^{\infty} f_{2}(s) \Delta s=\frac{1}{2} \lim _{A \rightarrow \infty} \int_{n_{0}}^{A} e^{-s-1} \Delta s=\frac{e^{-n_{0}}}{2(e-1)}<\frac{1}{e-1} .
\end{gathered}
$$

Then (4.6), (4.8), and (4.9), Proposition 1.6 and its exact analogues for the improper integrals of second kind imply that improper integrals $I_{1}$ and $I_{2}$ are convergent, and so

$$
\int_{0}^{\infty} e^{-\sigma(s)} G(\sigma(s), s) m(s) \Delta s \leq \frac{1}{2}\left(I_{1}+I_{2}\right) \leq \frac{1}{2}\left\{\int_{0}^{n_{0}} f_{2}(s) \Delta s+\int_{n_{0}}^{\infty} f_{2}(s) \Delta s\right\} \leq \frac{1}{e-1} .
$$


12 Singular boundary value problem on infinite time scale

Hence the condition $\left(\mathrm{H}_{3}\right)$ of Theorem 2.2 is fulfilled. So Theorem 2.2 gives us the desired conclusion.

\section{Acknowledgments}

The first author acknowledges support from USTC (KD2004002), NSFC (10471075), and NSF (Y2003A01). The second and third authors acknowledge support from CAS, EMC, and NSFC.

\section{References}

[1] R. P. Agarwal, M. Bohner, and W.-T. Li, Nonoscillation and Oscillation: Theory for Functional Differential Equations, Monographs and Textbooks in Pure and Applied Mathematics, vol. 267, Marcel Dekker, New York, 2004.

[2] R. P. Agarwal, M. Bohner, and D. O'Regan, Time scale boundary value problems on infinite intervals, Journal of Computational and Applied Mathematics 141 (2002), no. 1-2, 27-34.

[3] D. R. Anderson, Eigenvalue intervals for a two-point boundary value problem on a measure chain, Journal of Computational and Applied Mathematics 141 (2002), no. 1-2, 57-64.

[4] B. Aulbach and S. Hilger, Linear dynamic processes with inhomogeneous time scale, Nonlinear Dynamics and Quantum Dynamical Systems (Gaussig, 1990), Math. Res., vol. 59, Akademie, Berlin, 1990.

[5] M. Bohner and A. Peterson (eds.), Advances in Dynamic Equations on Time Scales, Birkhäuser, Massachusetts, 2003.

[6] C. J. Chyan and J. Henderson, Eigenvalue problems for nonlinear differential equations on a measure chain, Journal of Mathematical Analysis and Applications 245 (2000), no. 2, 547-559.

[7] J. J. DaCunha, J. M. Davis, and P. K. Singh, Existence results for singular three point boundary value problems on time scales, Journal of Mathematical Analysis and Applications 295 (2004), no. 2, 378-391.

[8] J. M. Davis, J. Henderson, K. R. Prasad, and W. K. C. Yin, Eigenvalue intervals for nonlinear right focal problems, Applicable Analysis. An International Journal 74 (2000), no. 1-2, 215-231.

[9] P. W. Eloe and J. Henderson, Positive solutions and nonlinear $(k, n-k)$ conjugate eigenvalue problems, Differential Equations and Dynamical Systems. An International Journal for Theory and Applications 6 (1998), no. 3, 309-317.

[10] L. H. Erbe and A. Peterson, Green's functions and comparison theorems for differential equations on measure chains, Dynamics of Continuous, Discrete and Impulsive Systems. An International Journal for Theory and Applications 6 (1999), no. 1, 121-137.

[11] Eigenvalue conditions and positive solutions, Journal of Difference Equations and Applications 6 (2000), no. 2, 165-191.

[12] _ Positive solutions for a nonlinear differential equation on a measure chain, Mathematical and Computer Modelling 32 (2000), no. 5-6, 571-585.

[13] L. H. Erbe and H. Y. Wang, On the existence of positive solutions of ordinary differential equations, Proceedings of the American Mathematical Society 120 (1994), no. 3, 743-748.

[14] Z. C. Hao, J. Liang, and T. J. Xiao, Positive solutions of operator equations on half-line, Journal of Mathematical Analysis and Applications 314 (2006), no. 2, 423-435.

[15] J. Henderson, A. Peterson, and C. C. Tisdell, On the existence and uniqueness of solutions to boundary value problems on time scales, Advances in Difference Equations 2004 (2004), no. 2, 93-109.

[16] J. Henderson and H. Y. Wang, Positive solutions for nonlinear eigenvalue problems, Journal of Mathematical Analysis and Applications 208 (1997), no. 1, 252-259. 
[17] S. Hilger, Analysis on measure chains - a unified approach to continuous and discrete calculus, Results in Mathematics. Resultate der Mathematik 18 (1990), no. 1-2, 18-56.

[18] C.-H. Hong and C.-C. Yeh, Positive solutions for eigenvalue problems on a measure chain, Nonlinear Analysis. Theory, Methods \& Applications. An International Multidisciplinary Journal. Series A: Theory and Methods 51 (2002), no. 3, 499-507.

[19] V. Lakshmikantham, S. Sivasundaram, and B. Kaymakcalan, Dynamic Systems on Measure Chains, Mathematics and Its Applications, vol. 370, Kluwer Academic, Dordrecht, 1996.

[20] W.-C. Lian, F.-H. Wong, and C.-C. Yeh, On the existence of positive solutions of nonlinear second order differential equations, Proceedings of the American Mathematical Society 124 (1996), no. 4, $1117-1126$.

[21] J. Liang, T.-J. Xiao, and Z.-C. Hao, Positive solutions of singular differential equations on measure chains, Computers \& Mathematics with Applications. An International Journal 49 (2005), no. 56, 651-663.

[22] K. Zima, Sur l'existence des solutions d'une équation intégro-différentielle, Polska Akademia Nauk. Annales Polonici Mathematici 27 (1973), 181-187.

Zhao-Cai Hao: Department of Mathematics, University of Science and Technology of China, Hefei, Anhui 230026, China

Current address: Department of Mathematics, Qufu Normal University, Qufu,

Shandong 273165, China

E-mail address: zchjal@163.com

Jin Liang: Department of Mathematics, University of Science and Technology of China, Hefei, Anhui 230026, China

E-mail address: jliang@ustc.edu.cn

Ti-Jun Xiao: Department of Mathematics, University of Science and Technology of China, Hefei, Anhui 230026, China

E-mail address: xiaotj@ustc.edu.cn 


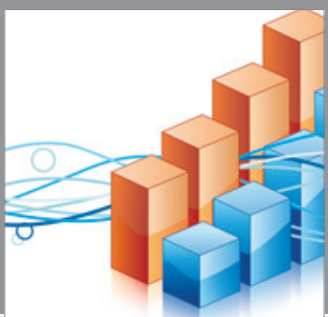

Advances in

Operations Research

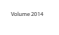

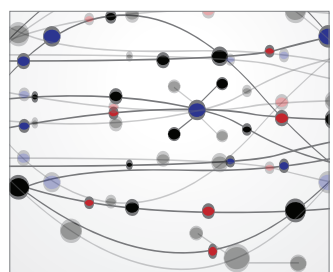

\section{The Scientific} World Journal
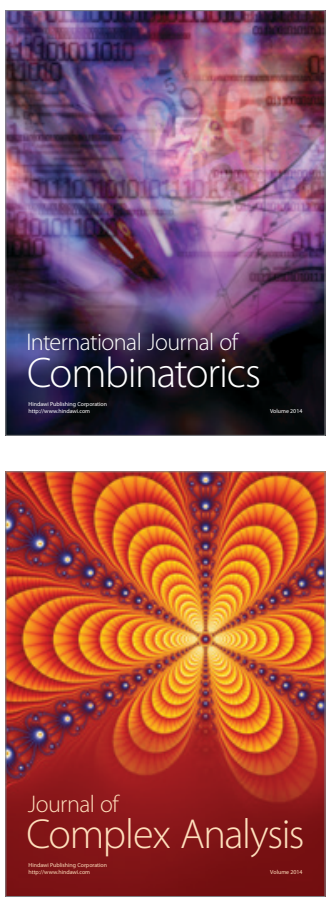

International Journal of

Mathematics and

Mathematical

Sciences
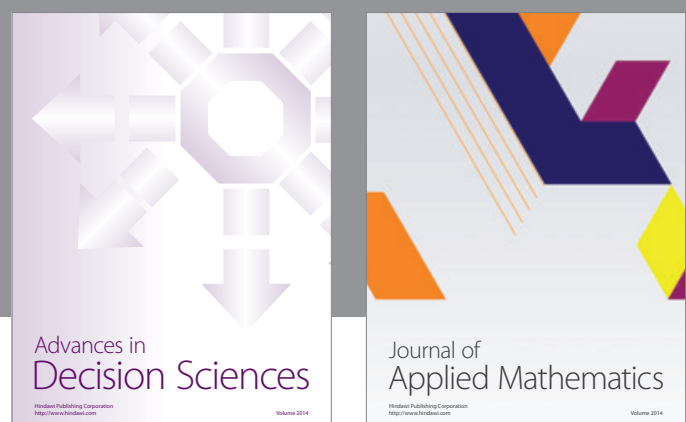

Journal of

Applied Mathematics
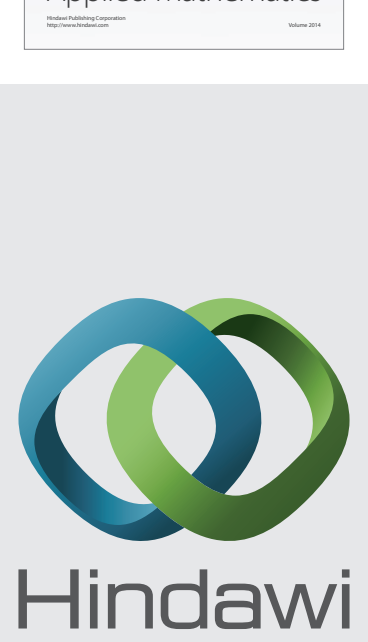

Submit your manuscripts at http://www.hindawi.com
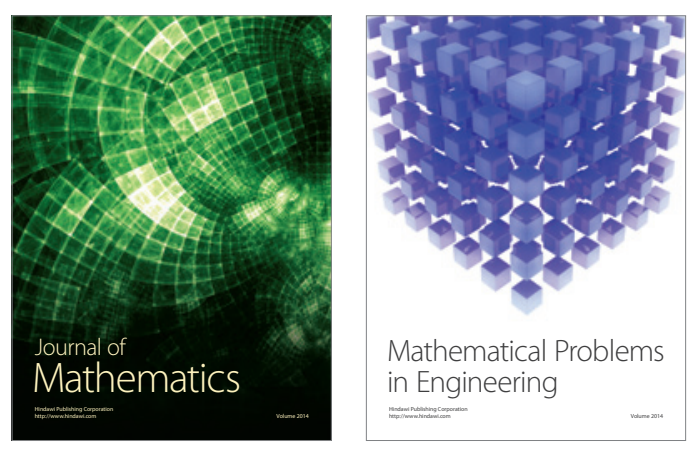

Mathematical Problems in Engineering
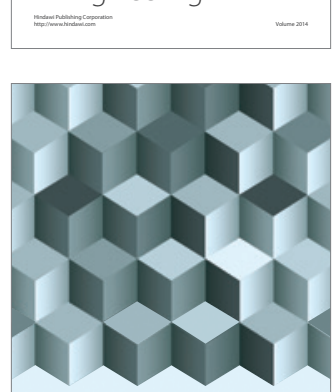

Journal of

Function Spaces
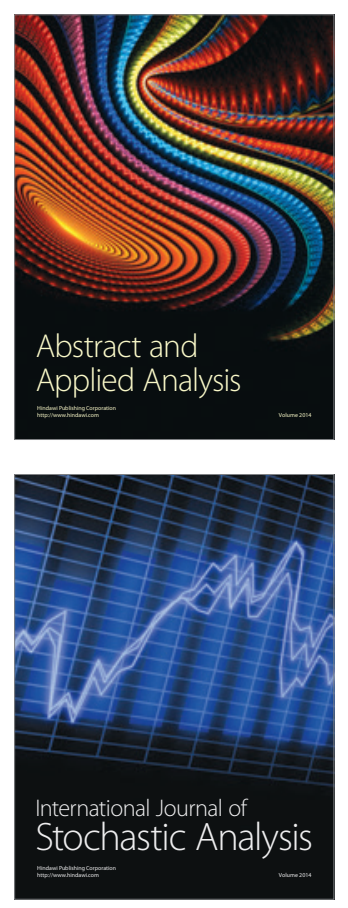

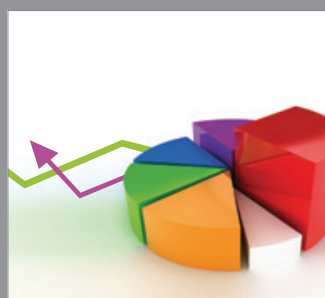

ournal of

Probability and Statistics

Promensencen
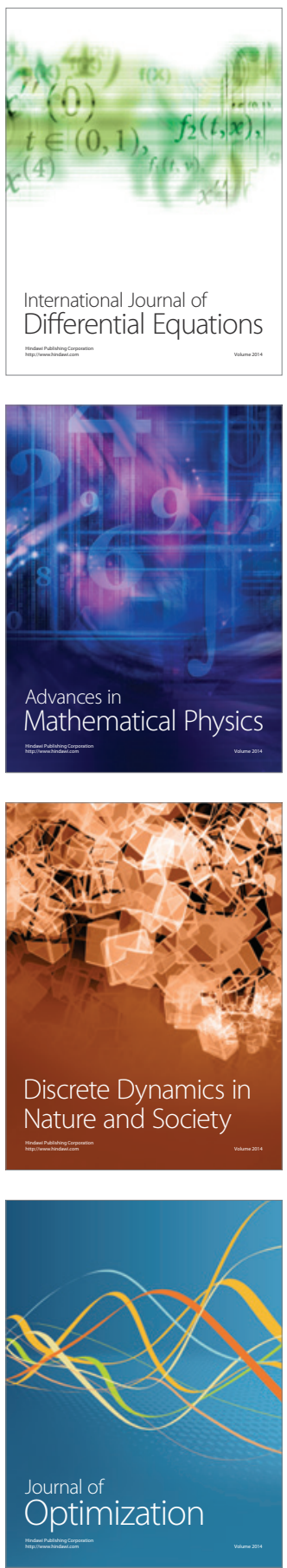